\title{
BAJA CALIFORNIA DENTRO DEL CONTEXTO DE LA MIGRACIÓN DE LA FRONTERA NORTE DE MÉXICO*
}

\author{
Por \\ Margarita Barajas Tinoco** \\ Adriana Wells Ayón**
}

\begin{abstract}
RESUMEN
En este trabajo se destaca el papel del fenómeno migratorio en la conformación poblacional de la frontera norte de México (FNM) en general y de Baja California en particular. Se describe el panorama de la inmigración interna a Baja California, haciendo especial énfasis en el perfil de la inmigración de la década de los ochenta, misma que se encuentra asociada con las oportunidades económicas de empleo que la entidad presentó en un periodo de crisis nacional. Las fuentes de información utilizadas son el Censo de Población, 1990, para Baja California y La Encuesta Continua de Migración de Baja California (ЕСMBC), 1989.
\end{abstract}

\begin{abstract}
This work emphasizes the role of the migration phenomenon in the population conformation of the northern border of Mexico (FNM) at large and in Baja California in particular. It describes the panoramic of the internal immigration to Baja California, making special emphasis in the outline of the immigration in the 80 's, which is found to be associated with the employment economic oportunities presented in this state during a period of national crisis.

The information sources used are: the population census of 1990 for Baja California, and the continuos migration poll of Baja California (ECMBC), 1989.
\end{abstract}

\section{INTRODUCCIÓN}

El presente trabajo intenta destacar cuál ha sido el papel de la migración interna en la conformación de una de las regiones más importantes del país: la frontera norte de México (FNM). ${ }^{1}$ Dentro de esta amplia zona que incluye seis estados colindantes con Estados Unidos (EE.UU.), se aborda

\footnotetext{
* Este documento es un producto del proyecto "Empleo en Baja California a partir del análisis de la PEA y de la movilidad geográfica general", que recibió apoyo de CONACYT. ** Investigadoras del Instituto de Investigaciones Sociales-UABC.

1 La región de la FNM a la que aquí se hace referencia la componen las seis entidades colindantes con EE.UU.: Baja California, Sonora, Chihuahua, Nuevo León, Coahuila y Tamaulipas.
} 
de forma particular el caso de Baja California. La relevancia de estudiar el fenómeno migratorio estriba en que amplía la comprensión sobre los procesos de poblamiento, según diferentes épocas.

Los procesos de población, como el de la migración, constituyen una manifestación más del desenvolvimiento de los niveles económicos y sociales peculiares en las respectivas configuraciones locales. En este sentido, el objetivo de este trabajo es exponer el fenómeno migratorio como proceso social, destacando su dinámica en la FNM.

Para el caso de Baja California interesa mostrar, además del comportamiento de la migración interna, el perfil de la población que alrededor del segundo quinquenio de la última década ha inmigrado a Baja California. Se trata de definir algunas asociaciones entre la población que en los últimos años ha tomado como residencia Baja California con la estructura de la fuerza de trabajo en la misma entidad. Por último, en las consideraciones finales se infieren algunas expectativas posibles en relación con la población inmigrante y su forma de incorporación a una estructura de trabajo.

\section{LA MIGRACIÓN COMO PROCESO SOCIAL}

La migración de manera general implica el traslado geográfico de personas a diferentes distancias y con distintas temporalidades (Standing, 1984). La importancia de este hecho radica en las implicaciones que en diversas dimensiones el fenómeno trae consigo, tanto en los lugares de origen, como en los lugares a los cuales se dirige el flujo. En otras palabras, la migración supone la existencia de dos espacios; el espacio geográfico dado por el desplazamiento físico en sí, y el espacio social, dado por la nueva estructura económica y su respectivo perfil en cuanto a sector de actividad, profesion, salario y condiciones de vida en el nuevo lugar. El supuesto latente en la conceptualización de espacio social es de que existen causas estructurales que impulsan a determinados grupos a ponerse en movimiento. Esas causas, casi invariablemente, son de fondo económico por desplazamiento de actividades en el espacio, y crecimiento diferencial de actividades en lugares distintos, alcanzando grupos que componen la estructura social del lugar de modo diferenciado (Gaudemar, 1979).

En el caso de la conformación de ciudades es definitorio el hecho, al menos para el caso latinoamericano, entre un crecimiento industrial capitalista y el proceso de migración rural-urbano, de aquí que se ha insistido en que el fenómeno de la migración, entendido como proceso, debe de ubicarse dentro del contexto de la industrialización, modelos de urbanización y el paso de una economía campesina a una economía de mercado 
(Singer, 1975), sin dejar de lado las políticas explícitas que desde el ámbito político y económico influyen de manera directa o indirecta. Por ejemplo, podríamos apuntar las implicaciones colaterales a una política de descentralización y desconcentración, ciertos convenios entre federación y estado en cuanto a inversión para impulsar alguna actividad económico-pruductiva, y tratamientos fiscales preferenciales con fines similares, entre otras.

\section{LA MIGRACIÓN COMO DINÁMICA DE CRECIMIENTO POBLACIONAL EN LA FRONTERA NORTE DE MÉXICO}

La región de la frontera norte de México (FNM) ha comportado dinámicas de crecimiento poblacionales muy distintas a las presentadas en otras regiones del país; mientras que por décadas fue una zona despoblada, actualmente en ella se encuentran varios centros poblacionales de importante jerarquía urbana que han mostrado tasas de crecimiento poblacionales superiores al promedio nacional, comparables con las tasas de los centros urbanos del país de acelerada expansión. Genéricamente, en la primera mitad del presente siglo el crecimiento de la zona estuvo asociado a los diferenciales en niveles de vida en la misma, y a la cercanía con un desarrollado país capitalista; simultáneo a esto deben considerarse los grandes proyectos de inversión pública dirigidos a alentar la actividad económica de la región a través de la introducción y mejoramiento de medios de comunicación terrestre y la política definida de desarrollar la zona mediante el Programa de Industrialización Fronteriza, puesto en marcha a mediados de la década de los sesenta.

Es ilustrativo apuntar que mientras que en 1940 la FNM contaba con 2.6 millones de personas, para 1990 tenía alrededor de 13.2 millones. El mayor crecimiento de esta zona se observó sobre todo entre 1940 y 1970 , periodo en el que se alcanzaron tasas de crecimiento anual de 7.9, 5.7 y 4.3 por ciento respectivamente; mientras que a nivel nacional se mantenía un promedio anual de 3.5 por ciento para el mismo periodo (CONAPO, 1988). Cabe señalar también que la proporción de población que guarda la FNM en el total nacional pas6 de constituir el $13.2 \%$ en 1940, a $16.3 \%$ en 1990. Esta dinámica poblacional se ha dado por dos componentes de crecimiento; por una parte el crecimiento natural, pero de manera relevante por el crecimiento social. De hecho, la población inmigrante, su intensidad y volumen, es lo que explica en buena medida el proceso de poblamiento y concentración en la zona. Con los resultados arrojados por el censo de población de 1990, se constata que las entidades que colindan con EE.UU., zon excepción de Coahuila, siguen presentando tasas de migración neta 
positivas, resaltando fundamentalmente en ese proceso, Baja California, Chihuahua y Tamaulipas; aunque hay que destacar que este aumento ha mostrado un mayor dinamismo en los municipios de la FNM que colindan con el país vecino. ${ }^{2}$ En efecto, mientras que en 1940 los municipios colindantes contabilizaban 383 mil habitantes, en 1990 alcanzaron casi los 3.9 millones de residentes. Esta cifra no se distribuye homogéneamente en el conjunto de los municipios contiguos, la concentración es particular sólo a algunos de ellos; se cuentan especialmente Ciudad Juárez en Chihuahua, Tijuana y Mexicali en Baja California y, Reynosa y Nuevo Laredo en Tamaulipas.

Baja California, como una entidad de la FNM ocupa un lugar muy importante ya que de los ocho municipios fronterizos más poblados, en esta entidad se encuentran los que ocupan el segundo y tercer lugar en tamaño poblacional. En cuanto a ritmos de crecimiento, Baja California también, comparativamente con el resto de las entidades de la FNM presenta tasas de crecimiento social mayores. La FNM, y en particular algunos de sus municipios fronterizos, han conformado una zona atractiva como punto de destino para importantes contigentes de población y como region de paso necesario para el país contiguo.

Las implicaciones del crecimiento poblacional por migración son diversas, por ejemplo, la presión sobre servicios públicos básicos, desempleo en determinadas actividades, provisión de recursos humanos para determinadas actividades productivas y de servicio, y otras. Si la provision se trata de mano de obra poco calificada, se esperaría que esto represente una ventaja para la economía del centro poblacional receptor que realiza una inversión mínima para la reproducción de la fuerza de trabajo. Fuera de estas posibilidades, lo que sí se puede apuntar es que dichas concentraciones han tenido un comportamiento dinámico y diverso en cuanto a oportunidad de tipo laboral, que se encuentra un déficit acumulado en infraestructura de considerable magnitud y que los problemas de transporte, comunicación y energía eléctrica comienzan a obstaculizar el optimo desarrollo de las actividades productivas. Esto, aunado a la no satisfacción de los servicios públicos elementales para importantes sectores de población, tienen una repercusión directa sobre la calidad de vida y bienestar de dichos grupos.

2 En 1990 INEGI publicó por primera vez tabuladores en conjunto para los municipios fronterizos de las seis entidades de la FNM; en dicha publicación están considerados 39 municipios como los que conforman la franja fronteriza (PF); tres en Baja California, once en Sonora, siete en Chihuahua, uno en Nuevo León y diez en Tamaulipas. Estos municipios están sobre 2,597 kilómetros, entre la línea divisoria internacional. 


\section{LA MIGRACIÓN INTERNA A BAJA CALIFORNIA}

La entidad ha sido un espacio geográfico en donde se han dado lugar distintos movimientos poblacionales provenientes de otras entidades del país, cuya verdadera importancia comienza a ser clave hacia finales de los años treinta y principios de los cuarenta, periodo en que se inicio un intenso desarrollo económico en el estado ${ }^{3}$ y que lo convertía esencialmente en punto de atracción para inmigrantes.

De esta manera, para la década de los cuarenta la población ya había aumentado en 30 veces más el total que tenía a principios del siglo, pues de alrededor de 7,500 habitantes en 1900, pas 6 a contar con cerca de 227,000, según el censo de 1950 (CONAPO,1984:5-10). A su vez, de 1900 a 1980 la población aumentó en 155.3 veces, al registrar el censo de 1980 un total de $1 ' 177,886$ habitantes. Comparativamente, este aumento significo dentro del total de la población nacional una mayor proporción, ya que de representar el $0.06 \%$ en 1900 , pasa al $1.76 \%$ en 1980 (CONAPO,1985:15) y al $2 \%$ en 1990 , lo cual indica un progresivo crecimiento de la población en la región.

Por otra parte, Baja California se distingue porque hasta 1940 su población aún no alcanzaba ni siquiera la cifra de 80 mil habitantes, lo cual significaba apenas un $3.0 \%$ del total de habitantes de la FNM. Sin embargo, ese rápido crecimiento poblacional que experimento después de 1940, le permite en 1990 , llegar a representar más del $12.0 \%$ del total de los habitantes de las seis entidades del norte de México.

El último censo de población y vivienda registra que en 1990 había 1'660,855 habitantes en Baja California; de los cuales un 47\%, declaro ser no nativa de la entidad, cabe señalar que este porcentaje es superior a los arrojados por los censos de $1970(41.6 \%)$ y $1980(46.3 \%)$, no obstante la baja manifestada en los flujos de inmigrantes a finales de los sesentas (cuadro 1).

Conforme al origen y dimension estructural de estos flujos según el lugar de nacimiento de los inmigrantes, a nivel estatal se observa que todavía a principios de los ochentas se presentaba una composición de la poblacion inmigrante constituida por dos corrientes: una proveniente de entidades vecinas (Sinaloa, Sonora y Baja California Sur) y otra de entidades distantes (Coahuila, Michoacán, Zacatecas y Jalisco) (CONAPO,1984:34). Para finales de los ochentas y principios de los noventas estas dos corrientes continúan presentándose, sin embargo, la primera de las corrientes se

3 Debido al aumento en la actividad agrícola, a la creciente demanda de trabajadores obreros, a la idustria en progreso con un buen abastecimiento de materia prima, y a la necesidad de más empleados y profesionistas para el gobierno, el comercio y la banca (CONAPO, 1984:11). 


\section{CUADRO 1. Baja California: Distribución de la población total nativa e inmigrante, $1970-1990$ (porcentajes).}

\begin{tabular}{lrrcr}
\hline Año & Total & Pob. nativa & Pob. inmigrante & No esp. \\
\hline 1970 & 100.0 & 58.4 & 41.6 & \\
1980 & 100.0 & 53.7 & 46.3 & \\
1988 & 100.0 & 56.9 & 43.1 & \\
1989 & 100.0 & 55.8 & 44.2 & \\
1990 & 100.0 & 50.8 & 47.0 & 2.2 \\
\hline
\end{tabular}

FUENTE: Censos generales de población de 1970, 1980, 1990. IIS-UABC y CONEPOBC con: Encuesta demográfica de Baja California 1986 (ED-BC,1986); Encuesta Continua de Población de Baja California 1988 (ECPBC,1988); y Encuesta Continua de Migración de Baja California 1989 (ECM-BC, 1989).

forma básicamente por población originaria de los estados de Sinaloa y Sonora; mientras que la segunda por originarios de Jalisco, Michoacán, y sumándose además el Distrito Federal (cuadro 2). En este sentido, de acuerdo con los datos obtenidos a partir de las fuentes de información consultadas, ${ }^{4}$ se puede afirmar que estas dos corrientes constituyen actualmente más de la mitad de la población inmigrante con residencia actual en Baja California.

A nivel municipal, resulta interesante encontrar que las distribuciones porcentuales de la población inmigrante en relación con la nativa y la distribución del origen de los flujos, se comporta de una manera particular en cada uno de los municipios de la entidad. En Ensenada el $44.1 \%$ de la población total resultó ser no nativa del municipio, en Mexicali el $36.7 \%$, en Tecate el $51.2 \%$, y en Tijuana el 56.0\%, resaltando este último como el porcentaje de población no nativa más alto (cuadro 3 ).

Cuando se analiza la población inmigrante por municipio, se observa que el comportamiento resulta un tanto diferente a como se da a nivel de la entidad, ya que si bien, los estados de origen de los flujos siguen siendo los mismos, el orden de importancia es distinto; mientras que para Ensenada, Técate y Tijuana, los residentes no nativos son preponderantemente

4 Encuesta demográfica de Baja Califomia, 1986 (resultados), Encuesta continua de población de Baja California (resultados 1988), Encuesta continua de migración de Baja Califormia de 1989 (resultados) y xI censo general de población y vivienda, 1990. 
CUADRO 2. Baja California: Distribución de la población total de inmigrantes residentes según lugar de nacimiento, 1986-1990 (porcentajes).

\begin{tabular}{lrrrr}
\hline Entidad federativa & 1986 & 1988 & 1989 & 1990 \\
\hline Total & 100.0 & 100.0 & 100.0 & 100.0 \\
Jalisco & 17.0 & 16.8 & 17.4 & 16.0 \\
Sinaloa & 11.0 & 10.0 & 14.6 & 13.8 \\
Michoacán & 9.0 & 7.2 & 10.9 & 9.3 \\
onora & 8.0 & 12.9 & 8.8 & 9.0 \\
'.F. & 6.0 & 6.6 & 6.7 & 7.4 \\
tras entidades & 43.0 & 46.5 & 41.6 & 44.5 \\
lo especificado & 6.0 & 0.0 & 0.0 & 0.0 \\
\hline
\end{tabular}

IENTE: ED-BC,1986; ECP-BC,1988; ECM-BC,1989; y XI censo general de población $y$ vivienda 1990, INEGI, México, 1990.

el estado de Jalisco, en Mexicali lo son de Sinaloa. Por otra parte, los ativos del estado de Oaxaca se concentran fundamentalmente como ctuales residentes en el municipio de Ensenada, de hecho constituyen más lel $11.0 \%$ de los inmigrantes a este municipio, mientras que en Mexicali 10 llega a ser ni el $1.0 \%$. Esto se explica, en parte, por la atracción que jercen ciertas actividades económicas, particularmente en el sector agrícola de Ensenada, que no logran satisfacerse con mano de obra local.

Aunque se registran residentes en todos los municipios de Baja California cuyo origen es el Distrito Federal, en términos relativos su importancia es mayor en Tijuana y Tecate, ya que este lugar de procedencia ocupa el cuarto orden dentro de los principales lugares de nacimiento de los inmigrantes a estos dos municipios.

En lo que se refiere a la distribución del total de inmigrantes a los municipios de Baja California, se puede observar que comparando las décadas 1970-1980 y 1980-1990, existen diferencias significativas, sobre todo en Tijuana y Mexicali, ya que por ejemplo entre $1970-1980,{ }^{5}$ el municipio de Tijuana contaba con el $45.06 \%$ de poblacion inmigrante residente en la entidad, y entre $1980-1990$ pasa a contar con el $60.52 \%$; es

5 Para la década 1970-1980, la distribución porcentual de la población inmigrante en B.C., era de $45.06 \%$ en Tijuana, $36.50 \%$ en Mexicali, $15.41 \%$ en Ensenada y de $3.03 \%$ en Tecate (CONAPO, 1984:224). 
CUADRO 3. Baja California: Distribución de la población total de inmigrantes y nativos por municipio, 1990.

\begin{tabular}{lccc}
\hline Municipio & Total & Nativos & Inmigrantes \\
\hline Ensenada & 100.0 & 55.9 & 44.1 \\
Mexicali & 100.0 & 63.3 & 36.7 \\
Tecate & 100.0 & 48.8 & 51.2 \\
Tijuana & 100.0 & 44.0 & 56.0 \\
\hline
\end{tabular}

FUENTE: XI censo general de población y vivienda 1990, INEGI, México, 1990.

decir, resulta notable el hecho de que actualmente la mayoría de los inmigrantes en el estado se encuentran residiendo en este municipio. Por su parte, llama también la atención el comportamiento del municipio de Mexicali, que de representar un $36.50 \%$ de la distribución entre 19701980 , disminuye su participación a un $21.69 \%$ en la siguiente década. En cuanto a los otros dos municipios: Ensenada y Tecate, la ditribución $1980-1990$ fue aproximadamente un $14.70 \%$ y $3.30 \%$ del total de inmigrantes, respectivamente (cuadro 4 ).

A partir del conocimiento de los antecedentes demográficos de Baja California, se confirma que el acelerado proceso de poblamiento que la caracterizo y que la coloca entre las principales entidades de México que se poblaron básicamente por crecimiento social, fue un elemento determinante de la actual conformacion poblacional. A nivel de los municipios lo que se constata es un cambio de participación en la proporción del total de inmigrantes según lugar de residencia, y un comportamiento diferente en

CUADRO 4. Baja California: Distribución de los inmigrantes a los diferentes municipios, entre los periodos 1970-1980 y 1980-1990 (porcentajes).

\begin{tabular}{lrr}
\hline Municipios & $1970-1980$ & $1980-1990$ \\
\hline Ensenada & 15.41 & 14.12 \\
Mexicali & 36.50 & 21.69 \\
Tecate & 3.03 & 3.65 \\
Tijuana & 45.06 & 60.52 \\
\hline
\end{tabular}

FUENTE: XI censo general de población y vivienda 1990, INEGI, México, 1990; y CONAPO, 1984:224. 
la magnitud y dirección de esa misma variable en relación con el municipio de destino o residencia actual.

\section{LOS INMIGRANTES INTERNOS DE LA DÉCADA DE LOS OCHENTA A BAJA CALIFORNIA}

En 1990, a partir de los datos arrojados por el último censo de población en la república mexicana, se encontró que Baja California ocupa el segundo lugar en la proporción de migración neta por cambio de lugar de residencia. La migración interna ${ }^{6}$ por sí misma es importante cuantitativamente hablando, en particular es en la década de los ochenta cuando los efectos de una crisis económica aguda hace estragos en el país, deteriorando los niveles de vida de la población, y aunque los efectos de la crisis se vivieron a nivel nacional, hubo zonas que por poseer ciertas ventajas comparativas se comportaron con menor vulnerabilidad; o más claramente, la crisis relativamente se resintio menos en razón de existir una serie de oportunidades económicas alternativas, como se cree que fue el caso de Baja California. La migración intercensal registrada entre 1980 y 1990 tuvo un aumento importante respecto de la registrada en la década anterior, 1970$1980{ }^{7}$ Esto nos habla pues de una reactivación de los flujos de inmigrantes ${ }^{8}$ en este periodo clave de la economía y procesos sociales del país.

Si observamos los datos de inmigración a Baja California en dos cohortes; $1980-85$ y $1985-90$, vemos que en el segundo quinquenio fue aún más importante este proceso, pues en el primero la tasa promedio anual de inmigración fue alrededor de un $3.7 \%$ y en el segundo de un $4.9 \%$, esta diferencia significa que el flujo inmigratorio pudo aumentar alrededor de un $32 \%$ en el segundo quinquenio de los ochentas, respecto al primero (cuadro 5). En este contexto un objetivo importante de este trabajo es proporcionar un perfil aproximado del grupo de inmigrantes que se recarga hacia el segundo quinquenio de los ochentas. Este objetivo se pretende cumplir con dos fuentes de información; a partir del Censo de Población de Baja California para 1990 y de la Encuesta Continua de Migración, 1989. La primer fuente da cuenta de los inmigrantes por cambio de lugar

\footnotetext{
6 Por ella se entiende a la población que desde otras entidades de México cambió su residencia habitual a Baja California.

Es importante señalar que los inmigrantes acumulados entre 1980-1990 fueron el equivalente a 2.5 veces los acumulados entre 1970-1980; el aumento es muy importante, aun tomando en cuenta la subestimación de la inmigración en 1980 debido al uso del concepto de "residente habitual", ampliamente analizada por Corona (1986).

8 En un trabajo reciente se identifica: "una patente y constante tendencia al incremento en las tasas de inmigración entre 1986 y 1989 , tanto a nivel del estado en su conjunto, como cada uno de los cuatro municipios de Baja California" (Estrella, 1991:52).
} 
de residencia, mayores de cinco años, acumulados en la entidad entre 1985 y la fecha de la aplicación del instrumento, marzo de 1990.; este tipo de información está dada a nivel de los municipios cruzada con el lugar de residencia anterior (entidad de procedencia) y sexo. Generalmente, la mayoría de las fuentes de información han permitido estimar los absolutos de las migraciones, pero cuando se requiere información más desglosada, por periodos o perfiles de los grupos, se tiene que recurrir a fuentes cuyos objetivos precisos son particularmente planteados para la comprensión del fenómeno migratorio, así, la segunda fuente que aquí se utiliza es la Encuesta Continua de Migración de Baja California (ECMBC) 1989, que brinda la posibilidad del manejo de un conjunto de variables sociodemográficas, respectivas a la población total que inmigró a Baja California entre 1984 y 1989 . Dichas variables se toman para definir el perfil propuesto.

\section{CUADRO 5. Baja California: Distribución total de los inmigrantes* del periodo 1980-1990 y su participación en dos quinquenios.}

\begin{tabular}{lrrrc}
\hline Lugar & \multicolumn{1}{c}{ 1980-1990 } & $\begin{array}{c}1980-1985 \\
\text { B }\end{array}$ & $\begin{array}{c}1985-1990 \\
\text { C }\end{array}$ & $\begin{array}{c}\text { Incremento } \\
\text { quinquenal } \\
\text { (C-B)/B }\end{array}$ \\
\hline Total & 434096 & 187038 & 247058 & $32 \%$ \\
Ensenada & 61297 & 25741 & 35556 & $38 \%$ \\
Mexicali & 94194 & 41698 & 52496 & $25 \%$ \\
Tecate & 15884 & 6118 & 9766 & $59 \%$ \\
Tijuana & 262721 & 113481 & 149240 & $31 \%$ \\
\hline
\end{tabular}

FUENTE: Censos generales de población y vivienda 1980 y 1990, INEGI, México.

* Incluye no especificado e inmigrantes de otro país.

Antes de exponer la información que se manejó para este trabajo, conviene señalar algunos puntos importantes.

- La información manejada permite dar cuenta de un perfil aproximado para una parte de la población inmigrante, ya que la descripción corresponde a los inmigrantes acumulados en cinco años, entre 1984 y 1989. En razón de que el impacto acumulado no permite metodologicamente diferenciar experiencias ocupacionales de diversos cohortes de migrantes que llegan en distintos momentos históricos, es una 
ventaja contar con una serie de información que podemos adjudicar a los inmigrantes de un periodo específico, la desventaja es de que no deja de ser un grupo acumulado, por tanto se advierte que:

- El perfil al que llegamos no puede ser generalizado indiscriminadamente en el tiempo, ya que los instrumentos de información particulares captan determinadas características de información para un grupo " $\mathrm{x}$ " en un momento " $\mathrm{x}$ "; las preguntas que captan las variables interrogan sobre un hecho presente (a excepción del lugar de residencia anterior), situaciones ocurridas o las expectativas de cualquier otra condicion quedan completamente al margen. Las trayectorias laborales de los grupos, que serían de fundamental importancia para dar cuenta de la adaptación de los inmigrantes al mercado de trabajo, no son posibles de conocer con el tipo de fuentes que usamos.

- La distribución de los inmigrantes según año de llegada no es homogénea.

- El flujo inmigrante sólo tiene un rasgo en común, su residencia actual en Baja California, por lo demás forman un grupo bastante heterogéneo en grados de escolaridad, experiencias laborales, grupo de edades, información del lugar de destino, año de llegada, etcétera.

- Aunque se establece un perfil único para todo el grupo de inmigrantes, se consideró importante identificar algunos razgos de subgrupos, por ejemplo, las actividades de una parte de la población económicamente inactiva (PEI), la escolaridad de los mismos y en razón de ello su posible incorporación al trabajo, y la "antigüedad" de todos los inmigrantes según años de residencia en la entidad.

- En este nivel del trabajo no se incursiona aún hacia el intento de establecer comparaciones entre una estructura ocupacional de migrantes y nativos, aunque se considera de particular interés. Antes de incursionar en tal objetivo se cree que es de suma importancia estudiar a la inmigración en sus particularidades, su dimension y magnitud precisamente en los ámbitos y tiempos donde ocurre.

\section{DESCRIPCIÓN DEL PERFIL}

Para 1990, a partir de la información censal, ha sido posible contabilizar el total de la población inmigrante 9 que dentro del periodo 1985-1990 decidió tomar como residencia habitual alguno de los

9 Con excepción de los menores de cinco años. 


\section{CUADRO 6. Baja California: Distribución de la población inmigrante* acumulada durante el periodo 1985-1990.}

\begin{tabular}{lrr}
\hline Lugar & \multicolumn{2}{c}{ Población inmigrante $1985-1990$} \\
\hline B.C. & 220848 & 100.00 \\
Ensenada & 31533 & 14.27 \\
Mexicali & 45007 & 20.37 \\
Tecate & 8398 & 3.8 \\
Tijuana & 135910 & 61.54 \\
\hline
\end{tabular}

FUENTE: XI censo general de población y vivienda 1990, INEGI, México, 1990.

* Segun lugar de residencia anterior (otra entidad).

cuatro municipios del estado de Baja California. El total de este flujo resulto de 220,848 inmigrantes que se distribuyeron de la siguiente manera: $61.5 \%$ en Tijuana, $20.3 \%$ en Mexicali, $14.2 \%$ en Ensenada y $3.8 \%$ en Tecate (cuadro 6).

De las entidades más importantes que expulsan población a Baja California, más de la mitad $(58.08 \%)$ proviene de Sinaloa, Jalisco, Distrito Federal, Sonora, y Michoacán de mayor a menor proporción; y de los estados de Guanajuato, Nayarit, Oaxaca, Estado de México, Durango, Puebla y Guerrero el $27 \%$; el resto de los inmigrantes provienen de las otras 20 entidades (cuadro 7 ).

En una variedad importante de estudios sobre migración, la predominancia en el sexo masculino ha sido el comportamiento típico; en el caso del grupo que ahora abordamos, el comportamiento es más o menos equilibrado, ya que el $51.6 \%$ correspondio al sexo masculino y el $48.3 \%$ al femenino (cuadro 8).

Utilizando información de la ECMBC, es posible ampliar el perfil del grupo observado, de esta manera, en lo que respecta a las edades en que se concentraba la población inmigrante durante el periodo en cuestion, se puede advertir a partir de esta segunda fuente que a nivel estatal, el $63.4 \%$ de los inmigrantes se acumulaba en solo tres grupos de edad; menores de 15 años (26.4\%), de 15 a 19 (17.2\%), y de 20 a 24 (19.8\%). A nivel municipal, Mexicali y Tijuana concentraron en estos dos últimos grupos el $62.6 \%$ y el $64.7 \%$ respectivamente; mientras que en Tecate se concentr6 el $62.3 \%$ y en Ensenada el $57.8 \%$. En este sentido se observa que en Tijuana se presenta una tendencia de estructura más joven en sus inmigrantes recientes (cuadro 9). 
CUADRO 7. Baja California: Población total por entidad de residencia anterior según lugar de residencia actual (1985-1990).

\begin{tabular}{|c|c|c|c|c|c|}
\hline \multirow{2}{*}{$\begin{array}{l}\text { Residencia } \\
\text { anterior }\end{array}$} & \multicolumn{5}{|c|}{ Lugar de residencia actual } \\
\hline & B.C. & Ensenada & Mexicali & Tecate & Tijuana \\
\hline Total & 220848 & 31533 & 45007 & 8398 & 135910 \\
\hline Ags. & 1304 & 117 & 268 & 55 & 864 \\
\hline B.C.S. & 3049 & 1456 & 608 & 99 & 886 \\
\hline Campeche & 275 & 49 & 76 & 7 & 143 \\
\hline Coahuila & 2047 & 245 & 450 & 96 & 1256 \\
\hline Colima & 2739 & 525 & 396 & 80 & 1738 \\
\hline Chiapas & 1317 & 231 & 182 & 45 & 859 \\
\hline Chihuahua & 2886 & 580 & 526 & 177 & 1603 \\
\hline D.F. & 25696 & 3475 & 3400 & 694 & 18127 \\
\hline Durango & 7935 & 628 & 965 & 678 & 5664 \\
\hline Guanajuato & 11603 & 1148 & 3217 & 405 & 6833 \\
\hline Guerrero & 5313 & 1057 & 529 & 95 & 3632 \\
\hline Hidalgo & 2041 & 126 & 233 & 82 & 2408 \\
\hline Jalisco & 25749 & 2776 & 4341 & 1093 & 17539 \\
\hline México & 8344 & 1060 & 1078 & 284 & 5922 \\
\hline Michoacán & 15874 & 1822 & 2992 & 819 & 10241 \\
\hline Morelos & 2993 & 272 & 231 & 82 & 2408 \\
\hline Nayarit & 11433 & 958 & 2184 & 294 & 7997 \\
\hline Nuevo León & 1211 & 202 & 295 & 68 & 646 \\
\hline Oaxaca & 8744 & 5253 & 672 & 175 & 2644 \\
\hline Puebla & 5692 & 492 & 392 & 178 & 4630 \\
\hline Querétaro & 1149 & 115 & 166 & 35 & 833 \\
\hline Quintana R. & 222 & 58 & 27 & 4 & 133 \\
\hline S.L.P. & 1294 & 129 & 215 & 64 & 886 \\
\hline Sinaloa & 39867 & 4309 & 11546 & 1278 & 22734 \\
\hline Sonora & 21097 & 2793 & 8022 & 925 & 9357 \\
\hline Tabasco & 381 & 66 & 105 & 12 & 198 \\
\hline Tamaulipas & 1078 & 180 & 333 & 22 & 543 \\
\hline Tlaxcala & 846 & 30 & 64 & 61 & 691 \\
\hline Veracruz & 4852 & 865 & 670 & 155 & 3162 \\
\hline Yucatán & 186 & 27 & 42 & & 117 \\
\hline Zacatecas & 3347 & 450 & 695 & 326 & 1876 \\
\hline $\begin{array}{l}\text { Entidades no } \\
\text { especificadas }\end{array}$ & 284 & 39 & 87 & 10 & 148 \\
\hline
\end{tabular}

FUENTE: XI censo general de población y vivienda 1990, INEGI, México, 1990.

* Según lugar de residencia anterior (otra entidad). 
CUADRO 8. Baja California: Distribución porcentual de inmigrantes por sexo (1985-1990).

\begin{tabular}{lcc}
\hline Lugar & Hombre & Mujer \\
\hline Total & 51.4 & 48.5 \\
Ensenada & 51.9 & 48.0 \\
Mexicali & 50.9 & 49.0 \\
Tecate & 52.6 & 47.3 \\
Tijuana & 51.4 & 48.5 \\
\hline
\end{tabular}

FUENTE: XI censo general de población y vivienda 1990, INEGI, México, 1990.

* Según lugar de residencia anterior (otra entidad).

CUADRO 9. Baja California: Distribución porcentual de la población inmigrante por municipio de residencia actual según grupos de edad (1984-1989).

Grupos de

Edad

B.C. Ensenada Mexicali Tecate Tijuana

\begin{tabular}{lrrrrr}
\hline Total & 100.0 & 100.0 & 100.0 & 100.0 & 100.0 \\
$0-4$ & 4.3 & 4.4 & 4.1 & 5.2 & 4.3 \\
$5-9$ & 11.3 & 14.4 & 11.8 & 13.9 & 10.4 \\
$10-14$ & 10.8 & 9.7 & 11.7 & 12.8 & 10.5 \\
$15-19$ & 17.2 & 9.8 & 13.2 & 14.7 & 20.2 \\
$20-24$ & 19.8 & 19.5 & 21.8 & 15.7 & 19.3 \\
$25-29$ & 12.4 & 13.6 & 14.5 & 9.4 & 11.5 \\
$30-34$ & 6.0 & 7.2 & 5.3 & 8.2 & 6.0 \\
$35-39$ & 5.7 & 6.7 & 5.0 & 5.3 & 5.9 \\
$40-44$ & 3.9 & 3.1 & 3.1 & 3.9 & 4.4 \\
$45-49$ & 2.3 & 1.7 & 3.2 & 2.2 & 2.1 \\
$50-54$ & 1.9 & 1.6 & 2.6 & 2.6 & 1.6 \\
$55-59$ & 1.2 & 1.0 & 2.0 & .9 & 1.0 \\
$60-64$ & .7 & 1.5 & .4 & 1.0 & .6 \\
65 y más & 1.9 & 5.8 & 1.4 & 3.5 & 1.4 \\
No especificado & .5 & & & .6 & .8 \\
\hline
\end{tabular}

FUENTE: ECM-BC, 1989. 
En cuanto a la condicion de alfabetismo, a partir de los inmigrantes mayores de seis años y más, se encontró que el $90.0 \%$ del total, sabe leer, $y$, en donde se concentra un poco más el indicador de analfabetismo, es en Ensenada (14.1\%) (cuadro 10). A partir de los niveles de escolaridad se conformaron tres amplios rangos, el primero correspondiente a preescolar, primaria y técnico con primaria incluyó a la mitad del grupo alfabeta (50.1\%); dentro del segundo nivel (secundaria, técnico con secundaria y preparatoria), se incluy 6 el $38.1 \%$. El tercer nivel, que va de técnico con primaria a posgrado, se concentró el $8.3 \%$. A nivel municipal, llama la atención que en Mexicali sólo se concentraron en el primer nivel $44.1 \%$, lo cual puede llegar a indicar relativamente más altos niveles de escolaridad en los inmigrantes de este municipio, además, este mismo municipio, junto con Ensenada, presentaron mayor importancia relativa en el tercer nivel de escolaridad, en otras palabras, respecto a los totales correspondientes a cada municipio, ellos reciben una alta proporción de inmigrantes más escolarizados (cuadros 11 y 12).

CUADRO 10. Baja California: Distribución porcentual de los inmigrantes mayores de 6 años de edad según condición de alfabetismo (1984-1989).

\begin{tabular}{lcccc}
\hline Municipios & Total & $\begin{array}{c}\text { Condición de alfabetismo } \\
\text { Sabe } \\
\text { leer (\%) }\end{array}$ & $\begin{array}{c}\text { No sabe } \\
\text { leer (\%) }\end{array}$ & N.S. \\
\hline Total & 100.0 & 89.6 & 10.2 & .2 \\
Ensenada & 100.0 & 85.9 & 14.1 & \\
Mexicali & 100.0 & 88.9 & 10.9 & .2 \\
Tecate & 100.0 & 91.7 & 8.1 & .2 \\
Tijuana & 100.0 & 90.3 & 9.4 & .3 \\
\hline
\end{tabular}

FUENTE: ECM-BC, 1989.

Caracterizando la incorporación de los inmigrantes de 12 años y más de edad a la estructura ocupacional, se observa lo siguiente: la proporción de la población económicamente activa (PEA) a nivel estatal correspondió el $61.3 \%$, y a nivel municipal se denotan dos niveles, de $50.6 \%$ en Mexicali $y$ de $65.6 \%$ en Tijuana. 
CUADRO 11. Baja California: Distribución porcentual de los inmigrantes de seis años y más de edad según nivel de escolaridad (1984-1989).

\begin{tabular}{lrrrrr} 
Nivel de & & & & & \\
Escolaridad & Total \% & Ensenada & Mexicali & Tecate & Tijuana \\
\hline Total & 100.0 & 100.0 & 100.0 & 100.0 & 100.0 \\
Sin estudios & 1.9 & 2.9 & 1.9 & 2.2 & 1.7 \\
Preescolar & 1.2 & .8 & 1.3 & 1.0 & 1.2 \\
Primaria & 48.4 & 53.0 & 41.5 & 57.2 & 49.8 \\
Tec. con prim. & .5 & & 1.3 & .4 & .2 \\
Secundaria & 27.8 & 17.5 & 27.6 & 21.6 & 29.7 \\
Tec. con sec. & 3.3 & 6.1 & 5.8 & 1.2 & 2.1 \\
Preparatoria & 7.0 & 6.0 & 9.5 & 8.5 & 6.1 \\
Tec. con prep. & 1.2 & .6 & .8 & 1.4 & 1.5 \\
Normal & .6 & .7 & .3 & .2 & .7 \\
Profesional & 6.5 & 8.0 & 7.7 & 4.7 & 5.9 \\
Posgrado & .4 & 1.7 & .6 & .2 & .2 \\
N.S. & 1.3 & 2.5 & 1.6 & 1.4 & .9 \\
\hline
\end{tabular}

FUENTE: ECM-BC, 1989.

CUADRO 12. Baja California: Distribución porcentual de inmigrantes de seis años y más de edad por grupos de escolaridad (1984-1989).

\begin{tabular}{lrrrrr}
$\begin{array}{l}\text { Nivel de } \\
\text { escolaridad }\end{array}$ & Total \% & Ensenada & Mexicali & Tecate & Tijuana \\
\hline Total & 100.0 & 100.0 & 100.0 & 100.0 & 100.0 \\
Sin estudios & 1.9 & 2.9 & 1.9 & 2.2 & 1.7 \\
1er. nivel & 50.1 & 53.8 & 44.1 & 58.6 & 51.2 \\
2do. nivel & 38.1 & 29.6 & 42.9 & 31.3 & 37.9 \\
3er. nivel & 8.7 & 11.0 & 9.4 & 6.5 & 8.3 \\
N.S. & 1.3 & 2.5 & 1.6 & 1.4 & .9 \\
\hline
\end{tabular}

FUENTE: ECM-BC, 1989.

1er nivel= preescolar, primaria, técnico con primaria.

2do. nivel= secundaria, técnico con secundaria, preparatoria.

3 er. nivel= técnico con preparatoria, normal, profesional y posgrado. 
Áhora bien, la proporción de la población ocupada respecto a la PEA, o bien tasa de ocupacion, resulto para el estado de $96 \%$, a nivel municipal el comportamiento más alejado de este indicador se dio en Mexicali con una tasa de ocupacion de $92.8 \%$, en correspondencia a este indicador es en este municipio donde la población inmigrante desocupada ${ }^{10}$ fue más importante, de $7.2 \%$ y los municipios más favorecidos en cuanto a altas tasas de ocupación fueron Tecate y Tijuana (cuadro 13).

Observando estos mismos indicadores por sexo se tiene lo siguiente: a nivel estatal las tres cuartas partes de la PEA está integrada por hombres y a nivel municipal el comportamiento es casi similar, excepto por Mexicali, donde los hombres constituyen el $80 \%$ de la PEA. La participación de las mujeres es más importante en Tijuana, municipio que a su vez tiene una diversificacion bastante amplia en las ramas correspondientes a comercio

\section{CUADRO 13. Baja California: Indicadores ocupacionales de la pobla- ción inmigrante mayor de 12 años y más de edad (1984- 1989).}

\begin{tabular}{lccll}
\hline Lugar & $\begin{array}{c}\text { TBP } \\
(1)\end{array}$ & $\begin{array}{c}\text { TNP } \\
(2)\end{array}$ & $\begin{array}{l}\text { TO } \\
(3)\end{array}$ & $\begin{array}{l}\text { TD } \\
(4)\end{array}$ \\
\hline B.C. & 49.5 & 61.3 & 96.0 & 4.0 \\
Ensenada & 44.6 & 58.2 & 66.3 & 3.7 \\
Mexicali & 39.0 & 50.6 & 92.8 & 7.2 \\
Tecate & 41.5 & 55.0 & 98.1 & 1.9 \\
Tijuana & 54.6 & 65.6 & 97.3 & 2.7 \\
\hline
\end{tabular}

FUENTE: ECM-BC, 1989.

TBP = Tasa bruta de participación: es el porcentaje que representa la población económicamente activa respecto a la población total.

TNP = Tasa neta de participación: es el porcentaje que representa la población económicamente activa respecto a la población de 12 años y más.

TO = Tasa de ocupación: es el porcentaje que representa la población ocupada respecto a la población económicamente activa.

TD = Tasa de desocupación: es el porcentaje que representa la población desocupada respecto a la población económicamente activa.

${ }^{10}$ Este grupo está constituido por personas correspondientes al grupo de la PEA que al momento de la encuesta se encontraban buscando trabajo. 
y servicios que a su vez favorecen la ocupación femenina. A nivel estatal, la tasa de ocupación masculina es un poco más alta que la correspondiente a mujeres; en Ensenada y Tijuana las tasas de ocupacion por sexo se mantienen a la par, incluso en Tijuana la tasa de ocupación femenina es levemente mayor que la de hombres (cuadro 14).

CUADRO 14. Baja California: indicadores de la población económicamente activa inmigrante por sexo (1984-1989).

\begin{tabular}{lccccc}
\hline Municipios & PEA & TBP & TNP & TO & TD \\
& & $(1)$ & $(2)$ & $(3)$ & $(4)$ \\
\hline
\end{tabular}

\begin{tabular}{|c|c|c|c|c|c|}
\hline Ensenada & 100.0 & & & & \\
\hline hombres & 73.0 & 72.59 & 86.38 & 97.84 & 2.1 \\
\hline mujeres & 27.0 & 21.49 & 30.48 & 92.19 & 7.8 \\
\hline Mexicali & 100.0 & & & & \\
\hline hombres & 81.0 & 64.31 & 83.41 & 93.10 & 6.89 \\
\hline mujeres & 19.0 & 15.18 & 18.80 & 91.30 & 8.69 \\
\hline Tecate & 100.0 & & & & \\
\hline hombres & 74.16 & 61.27 & 79.74 & 98.46 & 1.53 \\
\hline mujeres & 25.9 & 21.59 & 29.12 & 97.16 & 4.40 \\
\hline Tijuana & 100.0 & & & & \\
\hline hombres & 72.54 & 74.3 & 88.1 & 97.29 & 2.70 \\
\hline mujeres & 27.5 & 32.06 & 39.23 & 97.6 & 2.4 \\
\hline
\end{tabular}

FUENTE: ECM-BC, 1989.

TBP $=$ Tasa bruta de participación: es el porcentaje que representa la población económicamente activa respecto a la población total.

TNP = Tasa neta de participación: es el porcentaje que representa la población económicamente activa respecto a la población de 12 años y más.

TO = Tasa de ocupación: es el porcentaje que representa la población ocupada respecto a la población económicamente activa.

$\mathrm{TD}=$ Tasa de desocupación: es el porcentaje que representa la población desocupada respecto a la población económicamente activa. 
En cuanto a la inserción de los inmigrantes ocupados, es decir, población económicamente activa ocupada (PEAO) por sector de actividad económica, ${ }^{11}$ se tiene que el $54.7 \%$ se concentra en el sector III, el $32 \%$ y $11.6 \%$ en el II y I, respectivamente. Aunque las actividades económicas son diversificadas en todos los municipios, se denota predominancia de algunas de ellas en los mismos; en Ensenada por ejemplo, un importante número de sus inmigrantes (25.6\%) del periodo abordado se ocupa en la agricultura. La industria maquiladora resulta básica para los municipios de Mexicali y Tijuana, sobre todo en este último municipio, ya que del total de sus inmigrantes el $23.8 \%$ se acomoda en esta actividad. En Mexicali, los inmigrantes dentro de las actividades de comercio registran una proporción relativamente mayor respecto al resto de los municipios $(23.4 \%)$; en el caso de los servicios, es en Tijuana donde en mayor medida se acomoda el flujo de inmigrantes ocupados (cuadros 15 y 16).

CUADRO 15. Baja California; Distribución de la población inmigrante ocupada de 12 años y más de edad (PEAO), por municipio y sector de actividad económica (1984-1989).

\begin{tabular}{lrrrrr}
$\begin{array}{l}\text { Sector de } \\
\text { Actividad }\end{array}$ & Total & Ensenada & Mexicali & Tecate & Tijuana \\
\hline Total & 100.0 & 100.0 & 100.0 & 100.0 & 100.0 \\
I & 11.6 & 31.1 & 18.8 & 9.9 & 7.3 \\
II & 32.0 & 18.8 & 26.0 & 50.3 & 34.6 \\
III & 54.7 & 49.4 & 54.5 & 38.2 & 56.3 \\
N.E. & 1.7 & .7 & .8 & .8 & 2.1 \\
\hline
\end{tabular}

FUENTE: ECM-BC, 1989.

$\mathrm{I}=$ Agricultura, ganadería, y pesca.

II = Explotación de minas, industria de la transformación, industria maquiladora, industria de la construcción.

III = Energía eléctrica, comercio, transporte, gobierno, establecimientos financieros, turismo, educación, actividad doméstica, profesional, y otros.

N.E.= Actividades no especificadas.

\footnotetext{
"Se agrupan en tres sectores: el sector I (agricultura, ganadería y pesca); el sector II (explocación de minas, industria maquiladora, industria de la transformación e industria de la construcción); y el sector m (energía eléctrica, comercio, transporte, gobierno, establecimientos financieros, turismo, educación, actividades domésticas, profesionales y otros servicios).
} 
CUADRO 16. Baja California: Distribución porcentual de la población inmigrante ocupada de 12 años y más de edad (PEAO), según rama de actividad (1984-1989).

\begin{tabular}{|c|c|c|c|c|c|}
\hline $\begin{array}{l}\text { Rama de } \\
\text { actividad } \\
\text { económica }\end{array}$ & Total & Ensenada & Mexicali & Tecate & Tijuana \\
\hline Total & 100.0 & 100.0 & 100.0 & 100.0 & 100.0 \\
\hline $\begin{array}{l}\text { Agricultura y } \\
\text { ganadería }\end{array}$ & 10.8 & 25.6 & 17.9 & 7.6 & 7.2 \\
\hline Pesca & .7 & 5.5 & .9 & & \\
\hline Exp. minas & .1 & & & 2.3 & .1 \\
\hline Ind. transformación & 3.6 & & & 17.9 & 4.4 \\
\hline Ind.maquiladora & 20.4 & 7.1 & 14.1 & 22.3 & 23.8 \\
\hline Construcción & 8.0 & 11.7 & 11.9 & 10.1 & 6.4 \\
\hline Energía eléctrica & .1 & & .6 & & \\
\hline Comercio & 19.8 & 15.7 & 23.4 & 9.9 & 19.9 \\
\hline Transporte & 3.6 & & 1.5 & 1.8 & 4.7 \\
\hline Gobierno & 4.0 & 14.6 & 11.4 & 4.5 & .6 \\
\hline $\begin{array}{l}\text { Establecimientos } \\
\text { financieros }\end{array}$ & .2 & & . & & .3 \\
\hline Turismo & 4.3 & 3.3 & 1.6 & 3.2 & 5.2 \\
\hline Educación & .4 & 3.5 & & & .1 \\
\hline Doméstico & 2.0 & .5 & 3.3 & 1.4 & 1.9 \\
\hline $\begin{array}{l}\text { Técnicos y } \\
\text { profesionales }\end{array}$ & 5.0 & 11.0 & 4.5 & 3.2 & 4.4 \\
\hline Otros servicios & 15.3 & .8 & 8.2 & 14.2 & 19.2 \\
\hline No especificado & .7 & & & .5 & .9 \\
\hline N.S. & 1.0 & .7 & .8 & .3 & 1.2 \\
\hline
\end{tabular}

FUENTE: ECM-BC, 1989.

Respecto a la posición en el empleo de los inmigrantes correspondientes a la PEAO se puede observar que el rubro trabajadores a sueldo fijo, junto con el de trabajadores por su cuenta, concentraban a más del $87.9 \%$ del total en el estado. El comportamiento estatal lo representan de igual manera Mexicali, Tecate y Tijuana, no así Ensenada, donde la mayoría de sus inmigrantes se acumulaban en trabajadores a sueldo fijo y trabajadores a destajo $88.6 \%$ (cuadro 17 ). 
CUADRO 17. Baja California: Población inmigrante ocupada de 12 años y más de edad (PEAO), por posición en el trabajo y lugar de residencia actual (1984-1989).

\begin{tabular}{lrrrrr}
\hline & \multicolumn{5}{c}{ Población residente por municipio } \\
$\begin{array}{l}\text { Posición en } \\
\text { el trabajo }\end{array}$ & B.C. & Ensenada & Mexicali & Tecate & Tijuana \\
\hline Total & 100.0 & 100.0 & 100.0 & 100.0 & 100.0 \\
$\begin{array}{l}\text { Patrón } \\
\text { Subcontratista }\end{array}$ & .8 & & 1.8 & & \\
Trabaja & .4 & & & 1.1 & \\
$\begin{array}{l}\text { por su cuenta } \\
\text { A sueldo fijo }\end{array}$ & 12.1 & 9.1 & 8.7 & 11.6 & \\
$\begin{array}{l}\text { A destajo } \\
\text { Trab. familiar }\end{array}$ & 95.8 & 73.3 & 86.4 & 76.5 & 73.4 \\
Sin pago & .5 & 15.3 & 2.3 & 10.2 & 10.5 \\
No Especificado & 1.0 & 1.5 & & & \\
\hline
\end{tabular}

FUENTE: ECM-BC, 1989.

Profundizando un poco más sobre el grupo de personas que forman parte de la población económicamente inactiva (PEI), tenemos que la más importante proporción correspondi 6 a las personas dedicadas al hogar (70\%), seguida de los dedicados al estudio (25\%) (cuadro 18). Debido a que el grupo más importante correspondió a mujeres dedicadas a actividades del hogar, se estableció el perfil de escolaridad de las mismas para ver qué proporción correspondió al segundo nivel, encontrándose que el mismo representaba un $36.3 \%$ a nivel estatal y a nivel municipal la proporción más alta la mostró Mexicali con 49\% (cuadro 19); si recordamos que el segundo nivel incluye los rubros desde secundaria hasta preparatoria, pasando por técnicos con secundaria, se cree que al menos una parte importante de estas mujeres, son potencialmente oferta de mano de obra, al menos un nivel mínimo de escolaridad no sería el obstáculo.

\section{CONSIDERACIONES FINALES}

Si entendemos la migración como proceso social resulta coherente comprender que este fenomeno, observa una serie de cambios o manifestaciones; de hecho como proceso y como objeto de análisis, la migración ha sido uno de los fenómenos sociales más dinámicos y mayormente 
CUADRO 18. Baja California: Distribución de la población inmigrante económicamente inactiva por actividad de la semana pasada (1984-1989).

Actividad

Población no ocupada por municipio

de la semana B.C. Ensenada Mexicali Tecate Tijuana pasada

\begin{tabular}{lrrrrr}
\hline Total & 100.0 & 100.0 & 100.0 & 100.0 & 100.0 \\
$\begin{array}{l}\text { Estudios } \\
\begin{array}{l}\text { Quehacer del } \\
\text { hogar }\end{array}\end{array}$ & 25.0 & 19.7 & 23.8 & 30.4 & 26.8 \\
$\begin{array}{l}\text { Jubilado o } \\
\text { pensionado }\end{array}$ & 70.0 & 69.4 & 69.1 & 61.5 & 71.5 \\
$\begin{array}{l}\text { Vive de sus } \\
\text { rentas }\end{array}$ & 1.4 & .9 & 2.6 & 4.1 & .7 \\
$\begin{array}{l}\text { Incapacitado } \\
\text { permanente }\end{array}$ & 1.3 & 1.1 & & & \\
Otro & 1.7 & 9.0 & .8 & 2.2 & .1 \\
\hline
\end{tabular}

FUENTE: ECM-BC, 1989.

abordado desde distintas perspectivas teóricas. Si bien, el tipo de migración rural-urbana sigue manifestándose, se ha dado paso, entre otras variantes, al tipo de migración entre áreas urbanas. ${ }^{12} \mathrm{Al}$ respecto, se ha encontrado que existe una diversificación de las corrientes de migración en cuanto a mayor incorporación de regiones y menores grados de heterogeneidad en las mismas; así, el proceso social implícito en la migracion adquiere mayor complejidad por sus diversas modalidades y, seguramente también distintas implicaciones que requieren, a su vez, nuevas formas de explicación.

En cuanto al proceso de crecimiento poblacional de la FNM, en importante medida generado por crecimiento social, es importante anotar que más que pensar que la FNM y algunos de sus municipios fronterizos dejen

${ }^{12}$ En diversos estudios se ha encontrado que la población migrante cada vez en mayor medida selecciona menos a las ciudades primarias o más grandes como destino único, sino que se orienta más a las ciudades medias dentro de las propias regiones o a aquéllas que experimentan una dinámica sectorial particular (Cantú y Luque, 1990; Graizbord y Mina, 1993). 
CUADRO 19. Baja California: Distribución porcentual de mujeres inmigrantes del grupo PEI, dedicadas a actividades del hogar por grupos de escolaridad (1984-1989).

\begin{tabular}{lrrrrr}
$\begin{array}{l}\text { Nivel de } \\
\text { escolaridad }\end{array}$ & Total & Ensenada & Mexicali & Tecate & Tijuana \\
\hline Total & 100.0 & 100.0 & 100.0 & 100.0 & 100.0 \\
Sin estudios & 2.3 & 5.3 & 3.8 & 1.6 & .9 \\
ler. nivel & 55.2 & 59.4 & 40.8 & 71.3 & 61.4 \\
2do. nivel & 36.3 & 30.6 & 49.0 & 23.9 & 31.1 \\
3er. nivel & 3.9 & 4.7 & 4.3 & 2.3 & 3.8 \\
N.S. & .5 & 2.3 & .8 & & .5 \\
\hline
\end{tabular}

FUENTE: ECM-BC, 1989.

ler nivel $=$ preescolar, primaria, técnico con primaria . 2do. nivel $=$ secundaria, técnico con secundaria, preparatoria.

3er. nivel= técnico con preparatoria, normal, profesional y posgrado.

de crecer, se vislumbra que la región continuará apareciendo para muchos (migrantes, grupos de inversión, etcétera) un atractivo. ${ }^{13}$

De hecho, ante la política de globalización económica y del Tratado de Libre Comercio (TLC) se espera que la zona fronteriza expanda su base industrial y el conjunto de servicios correlacionados con el tipo de inserción económica que a la entidad corresponda en un nuevo modelo de inversión e intercambio comercial. Baja California, a su vez, se perfila dentro de este contexto como uno de los espacios más importantes de la FNM, en cuanto a su dinámica poblacional, jerarquía de sus ciudades e integración binacional en todas las dimensiones. La importancia de la inmigración interna a Baja California debe entenderse dentro del contexto nacional de redistribución de la población territorial que se registró más contundentemente a partir de los cincuenta, con el proceso de industrialización. Baja California fue uno de los ocho estados más importantes en cuanto a captador de inmigrantes entre 1950 y 1960 , este hecho se manifest 6 directamente en los indicadores de crecimiento social y natural que fueron tan dinámicos como los registrados en las principales metrópolis del país,

${ }^{13}$ Como apoyo a esto tenemos que los indicadores del PIB han sido más altos que el promedio nacional en esta zona, la captación bancaria favorable y generalmente bajos indices de desempleo a través del tiempo. 
por ejemplo el Distrito Federal (D.F.). En relación con la procedencia dt los inmigrantes resulta importante señalar que las corrientes básicas se han mantenido pero sumándose de manera ya importante el D.F., sobre todo a partir de la última década. A pesar de que en los ochentas se registraron en la entidad indicadores importantes sobre población emigrante, en 1990 Baja California continuó presentando los indicadores de migración neta más altos del país. Este hecho desde luego se mantiene relacionado, independientemente de las políticas de distribución poblacional, con el significado de las oportunidades económicas para los que realizan los desplazamientos. Respecto a la población que en la década de los ochenta inmigr6 a Baja California, se pueden señalar los siguientes puntos:

a) La preferencia de los inmigrantes por seleccionar un municipio particular (Tijuana) como punto de destino, conformando con ello el mayor nivel de concentración poblacional registrado por primera vez en un censo nacional y que coincide con tener el porcentaje más alto, junto con Tecate, de inmigrantes ocupados, en oposicion a Mexicali.

b) La relativa diversificación de "lugares anteriores de residencia" muy heterogéneas en cuanto a distancia, se tiene inmigrantes de entidades vecinas pero también distantes.

c) En cuanto a la selectividad por sexo no se encuentran concentraciones en uno u otro, en realidad no se altera el índice de masculinidad. ${ }^{14}$ Esto, aunado a la estructura básicamente joven (menores de 24 años) hace pensar que una parte importante de los inmigrantes de los ochenta lo hizo con el total de los integrantes familiares, indicador de ello es la alta proporción de inmigrantes menores de 15 años (26.4\%) aunque otra parte importante ha tenido cinco años para formarla en su nuevo lugar de residencia.

d) Se puso de manifiesto que en el segundo quinquenio de los ochentas se recargó el flujo inmigratorio de la década, y que además, el aumento fue dándose anualmente. En efecto, la distribución de inmigrantes según años de residencia en Baja California comportó una estructura de menor a mayor, desde $14.5 \%$ inmigrantes con cinco años de residencia, hasta la distribución más alta de un $26.5 \%$ inmigrantes de un año y menos de residencia (cuadro 20), este comportamiento mostrado en ascendencia constante nos induce a suponer que Baja California presentó una dinámica económica en expansión que pudo mantener una estructura diversificada de empleo, al menos hacia la conclusión de la década. Las demandas en servicio educativo por parte de los inmigrantes viene a ser muy importante,

${ }^{14}$ El índice de masculinidad se establece como la relación de hombres entre mujeres en una población definida. 
CUADRO 20. Baja California: Distribución porcentual del total de inmigrantes según periodo de llegada (1984-1989).

\begin{tabular}{lcrrrrr}
\hline $\begin{array}{l}\text { Periodo de } \\
\text { llegada }\end{array}$ & $\begin{array}{c}\text { Años de } \\
\text { residencia }\end{array}$ & Total & Ensenada & Mexicali & Tecate & Tijuana \\
\hline Total & & 100.0 & 100.0 & 100.0 & 100.0 & 100.0 \\
$1984 / 85$ & 5 años & 14.5 & 20.7 & 20.4 & 11.5 & 11.3 \\
$1985 / 86$ & 4 años & 17.0 & 10.0 & 12.0 & 17.0 & 20.1 \\
$1986 / 87$ & 3 años & 19.8 & 15.4 & 24.5 & 15.9 & 18.9 \\
$1987 / 88$ & 2 años & 22.2 & 22.9 & 20.8 & 25.8 & 22.4 \\
$1988 / 89$ & 12 meses & 26.5 & 31.0 & 22.3 & 29.8 & 27.2 \\
\hline
\end{tabular}

FUENTE: ECM-BC, 1989.

pues los menores de 15 años representaron más del 26\%. Por su parte, los mayores de 15 años, tienen en general una escolaridad relativamente alta, ya que alrededor del $47 \%$ tienen estudios de secundaria hacia arriba, son personas con los conocimientos mínimos necesarios para desenvolverse activamente en la región mientras exista demanda de empleo formal o demanda de sus servicios que como empleados o autoempleados (formal ylo informalmente) ofrezcan.

\section{BIBLIOGRAFÍA}

CANTÚ, Juan José y Rodolfo Luque G. 1990. "La migración a la zona metropolitana de la ciudad de México", DEMOS.

CONAPO. 1984. Estudio Sociodemográfico de Baja California, México, D.F.

- 1985. Baja California Demográfico, Breviario. México.

CONAPO. 1988. Demografía de la Frontera Norte de México.

CONEPO-BCUnstituto de Investigaciones Sociales-UABC. 1987. Encuesta Demográfica de Baja California, 1986 (resultados), Mexicali, B.C.

- 1989. Encuesta Continua de Población de Baja California, 1988 (resultados). Mexicali B.C.

- 1990. Encuesta Continua de Migración de Baja California, 1989 (resultados), Mexicali B. C.

CORONA, Rodolfo. 1986. "Evaluación de los datos censales de 1980". Población residente y migración en Baja California. Cuadernos 
CEFNOMEX. Centro de estudios fronterizos del Norte de México, Tijuana, Baja California.

ESTRELLA, V. Gabriel. 1991. "Dinámica de los componentes demográficos de Baja California" en Estudios Fronterizos, IIS-UABC, no. 26, septiembre-diciembre.

GAUDEMAR, Jean Paul. 1979. Movilidad del trabajo y acumulación del capital, ed. Era. México, 1ra. ed.

GRAIZBORD Boriz y Alejandro Mina. 1993. "Cambios en la relación población-territorio en México". Memorias de la IV Conferencia Latinoamericana de Población, Vol. II. INEGI-IIS-UNAM.

INEGI, XI censo general de población y vivienda de Baja California, 1990

INEGI, XI censo general de población y vivienda, frontera norte, 1990. INEGI.

SINGER, Paul. 1975. "Migraciones internas: consideraciones teóricas sobre su estudio", en Economía política de la urbanización. Ed. Siglo XXI, México.

STANDING, Guy. 1984. "Conceptualing Territorial Mobility", en R.E. Bilsborrow. Migration Surveys in Low-income Countries. Com Helm, Baja California dentro del contexto de la migración de la frontera norte de México 